\title{
Number Type
}

National Cancer Institute

\section{Source}

National Cancer Institute. Number Type. NCI Thesaurus. Code C62438.

Categories into which numbers are apportioned by the conventions of algebraic number theory. 